\title{
Semi-guided plane wave reflection by thin-film transitions for angled incidence
}

\author{
Fehmi Çivitci, Manfred Hammet*, Hugo J.W.M. Hoekstra \\ University of Twente, Enschede, The Netherlands
}

\begin{abstract}
The non-normal incidence of semi-guided plane waves on step-like or tapered transitions between thin film regions with different thicknesses, an early problem of integrated optics, is being reconsidered. As a step beyond the common effective index picture, we compare two approaches on how this problem can be tackled — at least approximately — by nowadays readily available simulation tools for integrated optics design.

Accepting the scalar approximation, using an ansatz of harmonic field dependence on the position along the interface, the 3-D problem reduces to a 2-D Helmholtz problem, for guided wave input and transparent-influx boundary conditions, with an effective permittivity that depends on the incidence angle.

Alternatively, one complements the structure with a second mirrored interface, such that the 2-D cross section of a wide multimode rib waveguide emerges. Constraints for transverse resonance then permit to translate the propagation constants of its polarized modes into discrete samples of the phase changes experienced by an in-plane guided wave upon total internal reflection at the sidewalls.
\end{abstract}

Keywords: integrated optics, slab waveguides, thin-film transitions, numerical/analytical modeling.

PACS codes: 42.82.-m 42.82.Bq 42.82.Et 42.82.Gw 42.15.-i

\section{Introduction}

Classical concepts [1, 2] for integrated optical components like lenses [3, 4], mirrors [5], prisms [6], but also for complex lens-systems [7], or, more recently, for entire spectrometers [8, 9], rely on the effects that a transition between regions with different layering has on thin-film guided, in-plane unguided light. Specifically this concerns tapered or step-like transitions between regions with different core thicknesses. Results for the reflection and refraction of 1-D guided plane waves at such a discontinuity may form the basis for a description of the in-plane propagation by geometrical optics [1, 2, 8]. Figure 1 provides a schematic view of the problem under consideration.

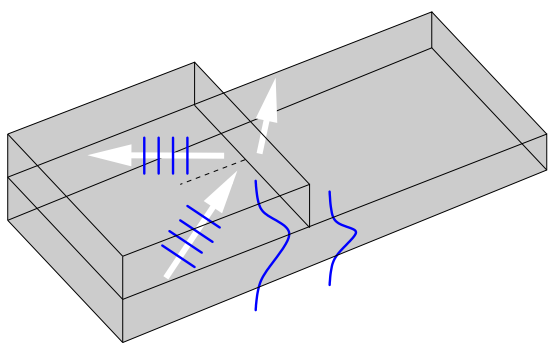

Figure 1: Incidence of vertically guided, laterally unguided plane waves under an angle on a step discontinuity between regions with different core film thicknesses. Primary interest is in the relative amplitude, and in the phase, of the reflected semi-guided wave, typically as a function of the angle of incidence. This phase change, or more precisely its angular derivative, determines the lateral displacement, the so-called Goos-Hänchen shift, of an in-plane-guided beam upon reflection at the transition [10, 11].

One might be tempted to reduce the actual 3-D problem of Figure 1 to two spatial dimensions by what is known as "effective index method" (cf. e.g. Ref. [12] and the references given therein), i.e. by representing the regions of different film thicknesses in terms of the effective modal indices of properly polarized slab waveguide modes, followed by applying the classical Fresnel-expressions [13] for reflection and transmission of plane waves under angled incidence. Unfortunately, this approach is highly questionable (radiation losses, ill-defined effective index [12]) even for the case of normal incidence, then a true 2-D problem. For angled incidence, one additionally neglects any effects due to the vectorial nature of the problem (wave hybridization).

As a starting point for further considerations, in Section 2 we briefly write out the exact equations. One arrives at a vectorial Helmholtz (-scattering) problem in two spatial dimensions, that is formally identical to the equations for the modes supported by waveguides with 2-D cross sections. For the scattering problem,

\footnotetext{
* $\mathrm{MESA}^{+}$Institute for Nanotechnology, University of Twente P.O. Box 217, 7500 AE Enschede, The Netherlands Phone: $+31 / 53 / 489-3448 \quad$ Fax: $+31 / 53 / 489-3996$ E-mail:m.hammer@utwente.nl
} 
a parameter, given by the incidence angle, takes the role of the propagation constant-eigenvalue in the mode problem. Furthermore, the boundary conditions for both problems differ. Nevertheless, in principle, it should be possible to re-use "half of" any vectorial code for eigenmode analysis, implement the proper parametrized transparent-influx-boundary conditions, and employ a solver for an inhomogeneous linear system in place of an eigenvalue solver, to arrive at an exact (numerical) solution of the problem in question. Such a line of action might be most convenient for those methods where the slab modes required for the specification of the incident waves play a role in the internal representation of the electromagnetic field anyway (Film-Mode-Matching [14], also available in a commercial context [15, 16]). Still, so far we did not come across any directly applicable simulation tools.

The task of Section 2 , however, resembles closely two types of problems for which software tools are readily available, namely solvers for scalar 2-D Helmholtz (scattering) -problems, with proper boundary conditions, on the one hand, and eigenmode solvers for dielectric channels on the other hand. It is the purpose of this paper to explore how far one can come by using these available tools, necessarily incurring certain approximations. The respective background is being outlined in Sections 3, 4, respectively. Section 3.2 relates to what might be understood as the Goos-Hänchen shift in the present context. Results are discussed and compared for a step discontinuity and for a series of tapered transitions in Section 5. This paper extends our preliminary account in Ref. [17].

\section{Formal problem}

We first look at a generic configuration as given schematically by Figure 2, Semi-guided plane waves, coming in from the $z$ - and $y$-homogeneous region (I), encounter an interface at $z=0$, or a transition region $z \geq 0$, respectively, which deviates from the slab profile in region (I).

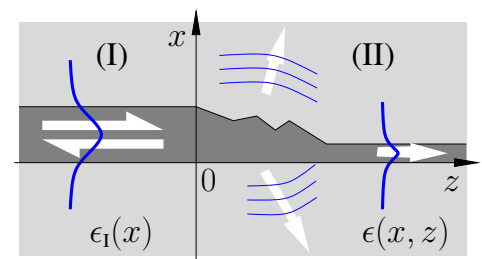

(a)

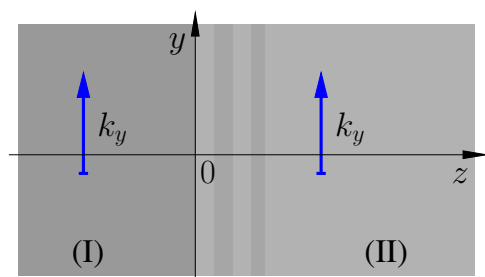

(b)

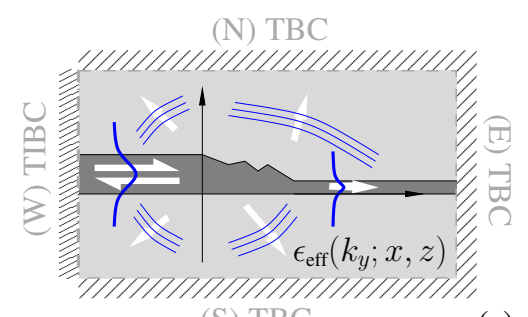

(c)

Figure 2: The actual 3-D configuration with cross sectional view (a) and top view (b) is being replaced by an effective problem (c) on a 2-D computational domain, fitted with transparent (influx) boundary conditions T(I)BCs.

The problem is governed by the homogeneous Maxwell equations in the frequency domain, for linear, isotropic, monmagnetic dielectric media:

$$
\operatorname{curl} \tilde{\boldsymbol{E}}=-\mathrm{i} \omega \mu_{0} \tilde{\boldsymbol{H}}, \quad \operatorname{curl} \tilde{\boldsymbol{H}}=\mathrm{i} \omega \epsilon \epsilon_{0} \tilde{\boldsymbol{E}} .
$$

The optical electric and magnetic fields $\tilde{\boldsymbol{E}}, \tilde{\boldsymbol{H}}$ oscillate in time $\sim \exp (\mathrm{i} \omega t)$ with frequency $\omega=k \mathrm{c}=2 \pi \mathrm{c} / \lambda$, for vacuum wavenumber $k$, wavelength $\lambda$, speed of light c, permittivity $\epsilon_{0}$, and permeability $\mu_{0}$. Structural information is given by the relative permittivity $\epsilon=n^{2}$, or by local values for the refractive index $n$.

Motivated by the $y$-homogeneity of the problem, $\partial_{y} \epsilon=0$, one looks for solutions of the form

$$
\left(\begin{array}{c}
\tilde{\boldsymbol{E}} \\
\tilde{\boldsymbol{H}}
\end{array}\right)(x, y, z)=\left(\begin{array}{c}
\boldsymbol{E} \\
\boldsymbol{H}
\end{array}\right)(x, z) \mathrm{e}^{-\mathrm{i} k_{y} y}
$$

where $k_{y}$ is a given parameter, typically related to the angle of incidence (cf. Section 3). After inserting this ansatz into the Maxwell equations (1), and after manipulations that eliminate four of the six components of $\boldsymbol{E}$ and $\boldsymbol{H}$, the remaining two components satisfy the equations

$$
\left(\begin{array}{cc}
\partial_{x} \frac{1}{\epsilon} \partial_{x} \epsilon+\partial_{z}^{2} & \partial_{x} \frac{1}{\epsilon} \partial_{z} \epsilon-\partial_{z} \partial_{x} \\
\partial_{z} \frac{1}{\epsilon} \partial_{x} \epsilon-\partial_{x} \partial_{z} & \partial_{x}^{2}+\partial_{z} \frac{1}{\epsilon} \partial_{z} \epsilon
\end{array}\right)\left(\begin{array}{c}
E_{x} \\
E_{z}
\end{array}\right)+k^{2} \epsilon_{\mathrm{eff}}\left(\begin{array}{c}
E_{x} \\
E_{z}
\end{array}\right)=0
$$


or, alternatively,

$$
\left(\begin{array}{cc}
\partial_{x}^{2}+\epsilon \partial_{z} \frac{1}{\epsilon} \partial_{z} & \partial_{x} \partial_{z}-\epsilon \partial_{z} \frac{1}{\epsilon} \partial_{x} \\
\partial_{z} \partial_{x}-\epsilon \partial_{x} \frac{1}{\epsilon} \partial_{z} & \epsilon \partial_{x} \frac{1}{\epsilon} \partial_{x}+\partial_{z}^{2}
\end{array}\right)\left(\begin{array}{c}
H_{x} \\
H_{z}
\end{array}\right)+k^{2} \epsilon_{\mathrm{eff}}\left(\begin{array}{c}
H_{x} \\
H_{z}
\end{array}\right)=0
$$

with

$$
\epsilon_{\mathrm{eff}}(x, z)=\epsilon(x, z)-k_{y}^{2} / k^{2} ;
$$

further equivalent variants exist. Formally these equations are identical to the vectorial equations for guided modes [11, 18], with the lateral wavenumber $k_{y}$ in the role of the propagation constant in the mode eigenproblem, here entering through the effective permittivity $\epsilon_{\mathrm{eff}}$.

For the specification of the incident waves, one observes that region (I) with permittivity $\epsilon_{\mathrm{I}}(x)$ represents a standard slab waveguide structure, characterized by $y$ - and $z$-homogeneity. Local solutions are transverse electric (TE) or transverse magnetic (TM) polarized separable planar waves of the form

$$
\begin{aligned}
& E(x, z)=\Psi(x) \mathrm{e}^{-\mathrm{i} \beta z}, \quad \partial_{x}^{2} \Psi+\left(k^{2} \epsilon_{\mathrm{I}}-\beta^{2}\right) \Psi=0, \quad \text { or } \\
& H(x, z)=\Psi(x) \mathrm{e}^{-\mathrm{i} \beta z}, \quad \epsilon_{\mathrm{I}} \partial_{x} \frac{1}{\epsilon_{\mathrm{I}}} \partial_{x} \Psi+\left(k^{2} \epsilon_{\mathrm{I}}-\beta^{2}\right) \Psi=0 .
\end{aligned}
$$

In order to represent the incoming guided slab mode, and also any reflected guided fields in region (I), the solutions of Eqs. (6), (7) for the principal electric or magnetic field components $E, H$ need to be complemented by expressions for the remaining five components of $\boldsymbol{E}$ and $\boldsymbol{H}$. The vectorial fields then need to be rotated according to the given angle of incidence.

One is left with a vectorial Helmholtz- (scattering-) problem on a 2-D computational domain (cf. Figure 2(c)), for the parametrized effective permittivity (5). The problem needs to be solved for boundary conditions that are transparent for outgoing guided and nonguided waves on all sides (N, W, E, S) of the computational domain, and that can accommodate the prescribed influx of a polarized vectorial plane guided wave, for the given angle of incidence $(\mathrm{W})$.

\section{Scalar theory}

For simplicity we first look at a step discontinuity, as illustrated schematically in Figure 3 . What follows relies on the approximation that, for the problem in question, polarization effects originating from interfaces or permittivity gradients can be disregarded. Neglecting the corresponding derivatives, the original Maxwell equations (1) turn into to the scalar Helmholtz equation

$$
\left(\partial_{x}^{2}+\partial_{y}^{2}+\partial_{z}^{2}\right) \tilde{E}+k^{2} \epsilon \tilde{E}=0 .
$$
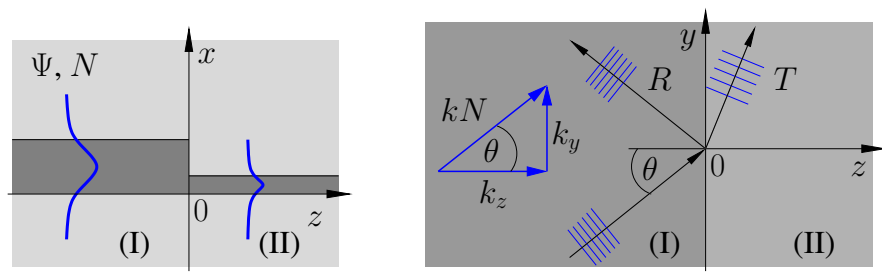

Figure 3: Step discontinuity, cross sectional and top views, with the relevant wave vectors and angles indicated.

As before, due to the homogeneity of the problem $\partial_{y} \epsilon=0$, one adopts an ansatz of harmonic $y$-dependence for the scalar field $\tilde{E}$ :

$$
\tilde{E}(x, y, z)=E(x, z) \mathrm{e}^{-\mathrm{i} k_{y} y} .
$$


In region (I), $z<0$, a slab mode with $x$-profile $\Psi$ and effective mode index $N$, nonguided in the $y$ - and $z$-directions, is supposed to be coming in at an angle $\theta$ :

$$
\tilde{E}_{\text {in }}(x, y, z)=\Psi(x) \mathrm{e}^{-\mathrm{i} k N(\sin \theta y+\cos \theta z)}, \quad \text { where } \quad \partial_{x}^{2} \Psi+k^{2}\left(\epsilon_{\mathrm{I}}-N^{2}\right) \Psi=0 .
$$

This incoming wave $\tilde{E}_{\text {in }}$ must satisfy the previous ansatz (9). Consequently, the lateral wavenumber $k_{y}$ has to be related to the angle of incidence as

$$
k_{y}=k N \sin \theta .
$$

One is left with the effective scalar problem

$$
\left(\partial_{x}^{2}+\partial_{z}^{2}\right) E+k^{2} \epsilon_{\mathrm{eff}} E=0, \quad \text { with } \quad \epsilon_{\mathrm{eff}}(x, z)=\epsilon(x, z)-N^{2} \sin ^{2} \theta
$$

which is to be solved on a 2-D computational domain with boundary conditions analogous to Figure 2(c), for the incoming field (10). Note that Eq. (12) emerges as well if one neglects the permittivity derivatives $(\partial \epsilon=\epsilon \partial)$ in the elements of the matrix operators of Eqs. (3), (4).

In principle, any suitable 2-D Helmholtz (scattering) solver for scalar TE fields could be applied. The outcome will be the field in the computational domain, or a numerical representation thereof, which, in region (I), can be given the form

$$
\tilde{E}_{\mathrm{I}}(x, y, z)=\left\{\Psi(x)\left(\mathrm{e}^{-\mathrm{i} k N \cos \theta z}+\rho \mathrm{e}^{\mathrm{i} k N \cos \theta z}\right)+\chi(x, z)\right\} \mathrm{e}^{-\mathrm{i} k N \sin \theta y},
$$

where $\chi$ is assumed to be orthogonal to the incoming profile, $\int \chi(x, z) \Psi(x) \mathrm{d} x=0$, for all $z<0$. This remainder represents any nonguided parts of the optical fields (radiation losses, backward propagating in region (I)) as well as guided waves of higher orders, if applicable. Our primary result is the complex reflection coefficient $\rho=\rho_{0} \mathrm{e}^{\mathrm{i} \phi}$ which separates into the reflectance $R=\rho_{0}^{2}$, and the phase change upon reflection $\phi$. In case guided modes are supported in region (II), also the guided wave transmittance $T$, defined analogously, might be of interest. Typically all quantities will be investigated as functions of the angle of incidence $\theta$.

\subsection{Total internal reflection}

Beyond the discontinuity, in the region (II) of Figure 3, the structure becomes $z$-homogeneous again. We assume that the core thickness there is smaller than the thickness in region (I), or, more precisely, that the effective index of the fundamental guided slab mode supported by region (II), if any, is smaller than $N$. Any in-plane propagating waves in region (II) can be characterized by a wavenumber $k n_{\mathrm{II}}$ and an effective index $n_{\mathrm{II}}$ that relate to the $y$-z-propagation. In case the layering of region (II) supports guided slab modes, the effective index of the fundamental mode constitutes an upper limit for $n_{\mathrm{II}}$. If no guided modes exist, the larger one of the refractive indices for $x \rightarrow \pm \infty$ in region (II) (i.e. the maximum of the substrate or cover refractive indices) establishes an upper bound for $n_{\mathrm{II}}$. Let $N_{\mathrm{II}}$ denote that limiting value.

Our ansatz (9) covers region (II) as well. All in-plane propagating waves there can thus be associated with a propagation angle $\theta_{\text {III }}$, such that Snell's law holds for the in-plane propagation:

$$
k_{y}=k N \sin \theta=k n_{\mathrm{II}} \sin \theta_{\mathrm{II}} \leq k N_{\mathrm{II}} \sin \theta_{\mathrm{II}} .
$$

Propagating waves in region (II) require $\sin \theta_{\text {II }}$ to be real, i.e. $\sin \theta_{\text {II }} \leq 1$. Consequently, if the angle of incidence $\theta$ exceeds the critical angle $\theta_{c}$ given by

$$
\sin \theta_{\mathrm{c}}=\frac{N_{\mathrm{II}}}{N}
$$

no in-plane propagating waves can exist in region (II), and hence no optical power is being transferred into that region (evanescent waves, which decay exponentially in the $+z$-direction, are well permitted). 
More formally this can be seen as follows. With a view to the completeness of the operator of Sturm-Liouville type in Eqs. (6), (10), here for region (II), we can restrict to solutions of Eq. (8), or Eq. (12), respectively, in the separable form

$$
E_{\mathrm{II}}(x, y, z)=\Psi_{\mathrm{II}}(x) \mathrm{e}^{-\mathrm{i}\left(k_{y} y+k_{\mathrm{II}, z} z\right)}
$$

where the local "mode" profile $\Psi_{\text {II }}$ satisfies

$$
\partial_{x}^{2} \Psi_{\mathrm{II}}+k^{2} \epsilon_{\mathrm{II}} \Psi_{\mathrm{II}}=\left(k_{y}^{2}+k_{\mathrm{II}, z}^{2}\right) \Psi_{\mathrm{II}} \leq k^{2} N_{\mathrm{II}}^{2} \Psi_{\mathrm{II}}
$$

Remember that $N_{\text {II }}$ has been introduced above as a limiting value, not (necessarily) the actual effective index of the wave in question. In-plane propagating waves of this form, i.e. solutions with $k_{\mathrm{II}, z}^{2} \geq 0$, thus require that $k^{2} N_{\mathrm{II}}^{2}-k_{y}^{2} \geq 0$, or, using the ansatz (11), that the angle of incidence $\theta$ does not exceed the critical angle (15).

A similar reasoning can be applied to any reflected waves in region (I) that propagate in the negative $z$-direction, i.e. that carry potential radiative losses. These waves can be associated with an effective index $N_{1}$, relating to $y$ - $z$-propagation. Typically $N_{1}$ would be the effective mode index of the first order slab mode, if applicable, or alternatively the maximum of the core/cladding refractive indices, as the limiting value for the continuum of core/cladding "modes". Also here we can associate an in-plane propagation angle $\theta_{1}$ with these waves, which needs to comply with Eq. (9):

$$
k_{y}=k N \sin \theta=k N_{1} \sin \theta_{1}
$$

Propagating waves in region (I), beyond the fundamental guided slab mode with effective index $N$, can exist only if $\sin \theta_{1} \leq 1$, or for incidence angles $\theta$ below the critical angle

$$
\sin \theta_{\mathrm{r}}=\frac{N_{1}}{N}
$$

for the in-plane propagation of higher order waves in region (I). In particular, for $\theta>\theta_{\mathrm{r}}$, no backwards traveling propagating waves are permitted in region (I), apart from the fundamental guided mode. Any optical power reflected from the interface is thus being carried away by that mode, there are no radiation losses due to reflected waves, the guided wave reflectance and transmittance (attributed to the fundamental mode only) add up to unity, $T+R=1$. If in addition $\theta>\theta_{\mathrm{c}}$, then the interface reflects the entire incident power into the backwards traveling fundamental guided mode, $R=1, T=0$. In that case one can indeed speak of total internal reflection for the semi-guided plane waves.

Note that, in case that regions (I) and (II) share the same $z$ - and $y$-uniform cladding, as considered in Section 5 . $\theta_{\mathrm{r}}$ also limits the range of incidence angles where higher order propagating waves can exist in region (II). In fact, as seen by evaluating Eq. (12) at a position where $\epsilon(x, z)=N_{1}^{2}$ (examples are the substrate and cover regions in the examples of Section [5), the effective permittivity $\epsilon_{\text {eff }}$ in the cladding is negative for incidence angles beyond $\theta_{\mathrm{r}}$, i.e. permits only $(x-, z-)$ evanescent waves. Note further that most of this reasoning applies as well for configurations with a tapered domain — of in principle arbitrary shape — in between the regions (I) and (II), as hinted at in Figure 2, Exceptions would be configurations with an intermediate higher refractive index, or larger core thickness (here one must expect the existence of guided waves, propagating in the $y$-direction), or large intermediate substrate or cladding indices that establish something like a half- or double-infinite "vertical core".

While these arguments rest on Eq. (8), i.e. are valid for scalar (TE-like) waves only, one could analogously write equations for a second set of characteristic angles that relate to waves with vertical $(-x)$ profiles of TM shape, satisfying Eq. (7). In case the transition / interface does not cause any (substantial) polarization coupling, the former considerations are applicable independently to TE- and to TM polarized waves. Otherwise one would have to consider both characteristic sets together in order to identify ranges of angles of incidence, where the different types of polarized waves in regions (I) or (II) can exist, i.e. where power transfer between the respective waves is permitted or forbidden. 


\subsection{Semi-guided beams}

Bundles of solutions (13), for different angles of incidence $\theta$, or different wavenumbers $k_{y}$, respectively, can describe what happens to a vertically guided, laterally wide, non-guided beam when it encounters the interface [10, 11]. For the guided part of the waves in region (I), and for configurations with total internal reflection $\left(\rho_{0}=1\right)$, such a superposition reads

$$
\tilde{E}_{\mathrm{I}, \mathrm{g}}(x, y, z)=\int A\left(k_{y}\right) \Psi(x)\left\{\mathrm{e}^{-\mathrm{i} k_{z}\left(k_{y}\right) z}+\mathrm{e}^{\mathrm{i} \phi\left(k_{y}\right)} \mathrm{e}^{\mathrm{i} k_{z}\left(k_{y}\right) z}\right\} \mathrm{e}^{-\mathrm{i} k_{y} y} \mathrm{~d} k_{y} .
$$

The second term represents the reflected waves; for total internal reflection only the phase part of the reflection coefficient remains. Its functional form $\phi\left(k_{y}\right)$ is known only implicitly through the numerical results. Note that the explicit dependence $k_{z}\left(k_{y}\right)$, as stated in Eq. (13), will not be used below.

We now assume that the amplitudes $A\left(k_{y}\right)$ of the wave packet are nonzero only in a small region of values $k_{y}$ around $k_{y 0}=k N \sin \theta_{0}$, related to the principal angle of incidence $\theta_{0}$ of the beam, such that expansions $k_{z}\left(k_{y}\right) \approx k_{z 0}+\left(k_{y}-k_{y 0}\right) v_{0}$ and $\phi\left(k_{y}\right) \approx \phi_{0}+\left(k_{y}-k_{y 0}\right) \Delta_{0}$ of first order, with abbreviations $k_{z 0}=k_{z}\left(k_{y 0}\right)$, $v_{0}=\left.\frac{\mathrm{d} k_{z}}{\mathrm{~d} k_{y}}\right|_{k_{y 0}}, \phi_{0}=\phi\left(k_{y 0}\right)$, and $\Delta_{0}=\left.\frac{\mathrm{d} \phi}{\mathrm{d} k_{y}}\right|_{k_{y 0}}$, suffice for evaluating the integrals formally:

$$
\tilde{E}_{\mathrm{I}, \mathrm{g}}(x, y, z)=\Psi(x)\left\{F\left(y+v_{0} z\right) \mathrm{e}^{-\mathrm{i} k_{z 0} z}+\mathrm{e}^{\mathrm{i} \phi_{0}} F\left(y-v_{0} z-\Delta_{0}\right) \mathrm{e}^{\mathrm{i} k_{z 0} z}\right\} \mathrm{e}^{-\mathrm{i} k_{y 0} y} .
$$

The incident and the reflected beam share the same envelope $F(\xi)=\int A\left(k_{y 0}+q\right) \mathrm{e}^{-\mathrm{i} \xi q} \mathrm{~d} q$, where, in the plane of incidence $z=0$, the reflected beam is displaced by the lateral distance $\Delta_{0}$, relative to the incident beam. Using the relation (11), and dropping the zero subscripts, the expression for the Goos-Hänchen shift $\Delta$ of a beam at incidence angle $\theta$ can be given the form

$$
\Delta=\frac{1}{k N \cos \theta} \frac{\mathrm{d} \phi}{\mathrm{d} \theta}
$$

According to the schematic view in Figure 4, the lateral shift can be viewed as a geometric reflection at an effective boundary at a distance $\delta=\Delta /(2 \tan \theta)$ behind the actual physical interface. Respective values for $\Delta$ and $\delta$ complement the results for reflectance $R$ and phase change upon reflection $\phi$ in Section 5. Note that the reasoning in this section remains valid for total internal reflection at slab waveguide transitions of arbitrary shape (e.g. for the tapered configurations of Section [5.2), as long as the region (I) on the left $z<0$ of the "interface" is $z$-homogeneous (where the precise interface position is arbitrary, in principle), provided that the data for $\phi(\theta)$ is calculated and applied consistently.

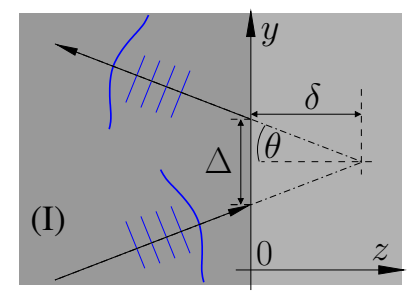

Figure 4: Lateral shift $\Delta$ (Goos-Hänchen-shift) of a semi-guided beam upon total internal reflection with incidence angle $\theta$ at the border of region (I). The displacement can be viewed as the effect of a geometric reflection of the ray associated with the beam at an effective interface that is positioned at a distance $\delta$ apart from plane $z=0$ of the physical discontinuity.

\section{Transverse resonance}

A standard dielectric rib waveguide can be viewed as being made from two of the former steps, separated by the width of the rib. Both types of structures share the invariance along the discontinuity / propagation coordinate, in our case the Cartesian $y$-axis. Hence, at least when restricting things to configurations with total internal reflection at the step discontinuity, one might expect that the properties of the guided modes supported by the rib are being determined by what happens at the - then two - step discontinuities that form the channel. Vice versa, one might expect that any known properties of guided modes might tell something about the sidewalls. Figure 5 illustrates this line of reasoning. Relevant here are fields of vertical $(x)$ fundamental order, in line with Section 3 for scalar TE or quasi-TE polarization. 


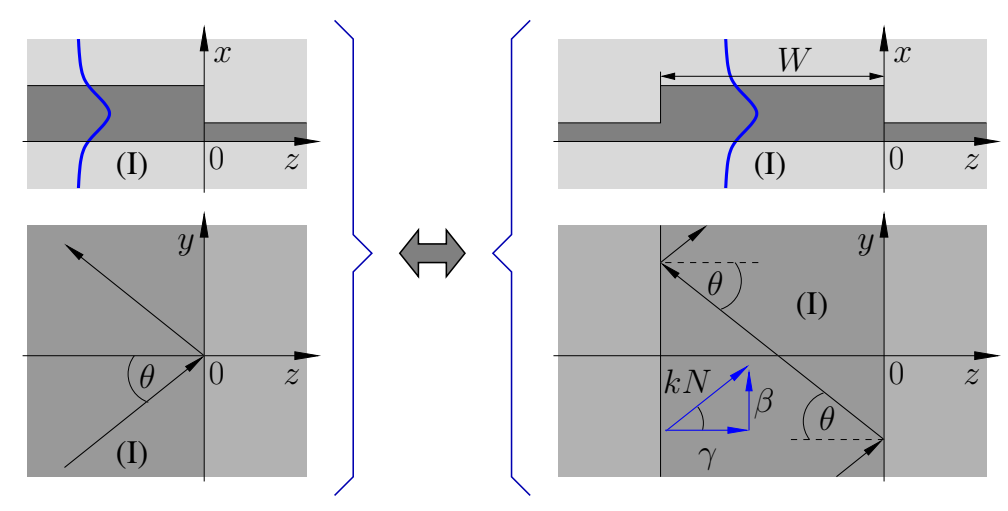

Figure 5: Two of the former step discontinuities, mirrored at the $x-y$-plane and placed at some distance, establish a waveguide configuration (2-D cross section) with rib width $W$.

In the core region $-W<z<0$, where $\epsilon(x, z)=\epsilon_{\mathrm{I}}(x)$, the principal component $E$ of the mode profile satisfies the equation

$$
\left(\partial_{x}^{2}+\partial_{z}^{2}\right) E+\left(k^{2} \epsilon_{\mathrm{I}}-\beta^{2}\right) E=0 .
$$

Accepting, for this central region, the approximation of a separable field $E(x, z)=\Psi(x) \zeta(z)$, where $\Psi$ is the 1-D TE mode of the central slab, $\partial_{x}^{2} \Psi+k^{2}\left(\epsilon_{\mathrm{I}}-N^{2}\right) \Psi=0$, one is led to a second 1-D slab mode problem for the lateral shape $\zeta$,

$$
\partial_{z}^{2} \zeta+\left(k^{2} N^{2}-\beta^{2}\right) \zeta=0
$$

with the solution $\zeta(z)=\zeta_{0} \mathrm{e}^{ \pm \mathrm{i} \gamma z}$ for $-W<z<0$, with $\gamma^{2}=k^{2} N^{2}-\beta^{2}$. The waves in the interior region are thus propagating with lateral wavenumber $\gamma=k N \cos \theta$, with propagation constant $\beta=k N \sin \theta$, and at a mode angle $\theta$ that can be interpreted as the angle of incidence of these interior waves on the rib sidewalls (cf. Figure 5). Values of $\beta$, and corresponding mode orders $m$, that relate to guided solutions are to be found as roots of the transverse resonance condition associated with the problem (24):

$$
-m 2 \pi=-W \gamma+\phi-W \gamma+\phi, \quad \text { or } \quad \phi=W k N \cos \theta-m \pi
$$

(note the sign convention adopted in Eqs. (1), (13) for the phase factors). Among other quantities the phase change $\phi$ of the waves upon reflection at the rib sidewalls is so far unknown.

Now presume that the guided modes of the channel, and a corresponding set of lateral mode indices and propagation constants, are available by means of some other existing suitable solver. If those modes are reasonably well approximated by our former ansatz of separable fields, with a lateral shape according to Eqs. (24), (25), we may use the transverse resonance condition as a means to determine $\phi$, by supplying the - now given values for the mode parameters $m$ and $\beta$. One obtains estimates for the phase change $\phi$ upon reflection at the rib sidewalls, for a series of sample values of incidence angles $\theta$ that relate to the set of discrete propagation constants $\beta$ through $\sin \theta=\beta /(k N)$. All modes are presumed to share the same $x$-profile $\Psi$; hence the distance $W$ determines the discrete values $\theta$. As long as the underlying approximations remain valid, one expects that results from mode solver runs for different (large, arbitrary) widths $W$ sample the same smooth curve $\phi(\theta)$.

Obviously this reasoning, in the present form, applies to guided modes only, i.e. to incidence angles $\theta$ larger than the critical angle for total internal reflection. Note that the assumption of separable fields in the center of the rib constitutes a quite restrictive approximation. Effects like mode hybridization at the sidewalls, and contributions from higher order slab modes or evanescent fields, are obviously disregarded. In many cases, however, typically for wide and shallow ribs, one obtains excellent results with quasi-TE (or -TM) approximations of — in principle fully vectorial — guided modes [19]. A similar accuracy can, presumably, be expected for the present analysis of comparable configurations.

In fact, one might view the fully vectorial mode analysis of comparable rib waveguides (in the sense of Figure 5) as some test for the theory of the present and previous sections. If the minor components of all guided modes are clearly negligible, then pronounced polarization coupling does not play a role for the configuration in question, i.e. the present scalar models should be adequate. We've found this to be the case for all structures considered in the next section. 


\section{Examples}

The numerical experiments of the following paragraphs rely on the quasianalytical scalar 2-D Helmholtz solver of [20, 21]. Guided mode analysis of channels with 2-D cross sections, as required for the approach of Section 4 , has been carried out with the quasianalytical technique of Ref. [22] (step discontinuities), and with the vectorial finite-difference solver of Ref. [16] (tapered transitions). Note that errors are inherent to all these results. For the Helmholtz solver, mainly the effect of the limited computational window and the staircase approximation, for the tapered solutions, are to be mentioned. The mode solvers are invoked for quasi-TE or vectorial, TElike polarized waves. Consequently, different continuity conditions for the lateral interfaces might cause a disagreement with the truly scalar results from the Helmholtz solvers. Although reasonable convergence, up to the scale of the figures, has been assured in all cases, certain "noisy" features in the respective curves, especially when it comes to derivatives (i.e. differences of possibly defective values), must probably still be attributed to numerical uncertainties.

Parameters have been adopted to be comparable with the practical design of the prism spectrometer in Ref. [9]. Referring to the insets of Figures 6, 8, configurations are specified in terms of the core and cladding refractive indices $n_{\mathrm{g}}=2.0081$ and $n_{\mathrm{b}}=1.4524$, the slab thicknesses in region (I) $d=160 \mathrm{~nm}$ and region (II) $r=40 \mathrm{~nm}$, for vacuum wavelength $\lambda=850 \mathrm{~nm}$, and in-plane (TE, quasi-TE) polarized (scalar) waves. The slabs of thicknesses $d$ and $r$, respectively, support guided modes with effective indices $N=1.678$ and $N_{\text {II }}=1.479$. Eq. (15) predicts a critical angle $\theta_{\mathrm{c}}=61.75^{\circ}$ for total internal reflection at the transitions. Radiation losses are forbidden for $\theta$ larger than the angle $\theta_{\mathrm{r}}=59.92^{\circ}$, in line with Eq. (19), where the cladding refractive index $n_{\mathrm{b}}$ has been supplied for $N_{1}$. Unless stated otherwise, these values apply to all simulations in Sections 5.1, 5.2,

\subsection{Step discontinuity}

Figures 6, 7 summarize our simulations for reflection at a step discontinuity. We look at the entire range of incidence angles first, by means of the scalar approach of Section 3 . According to Figure 6(a), the level of transmittance $T=0.74$, at normal incidence, remains nearly stationary for incidence angles close to $\theta_{\mathrm{r}}$. The level of reflectance, $R<0.01$ for $\theta=0$, increases gradually with $\theta$ approaching that limit. For $\theta \geq \theta_{\mathrm{r}}$ one indeed finds $R+T=1$; the transmittance drops to $T=0$ for $\theta \geq \theta_{\mathrm{c}}$.
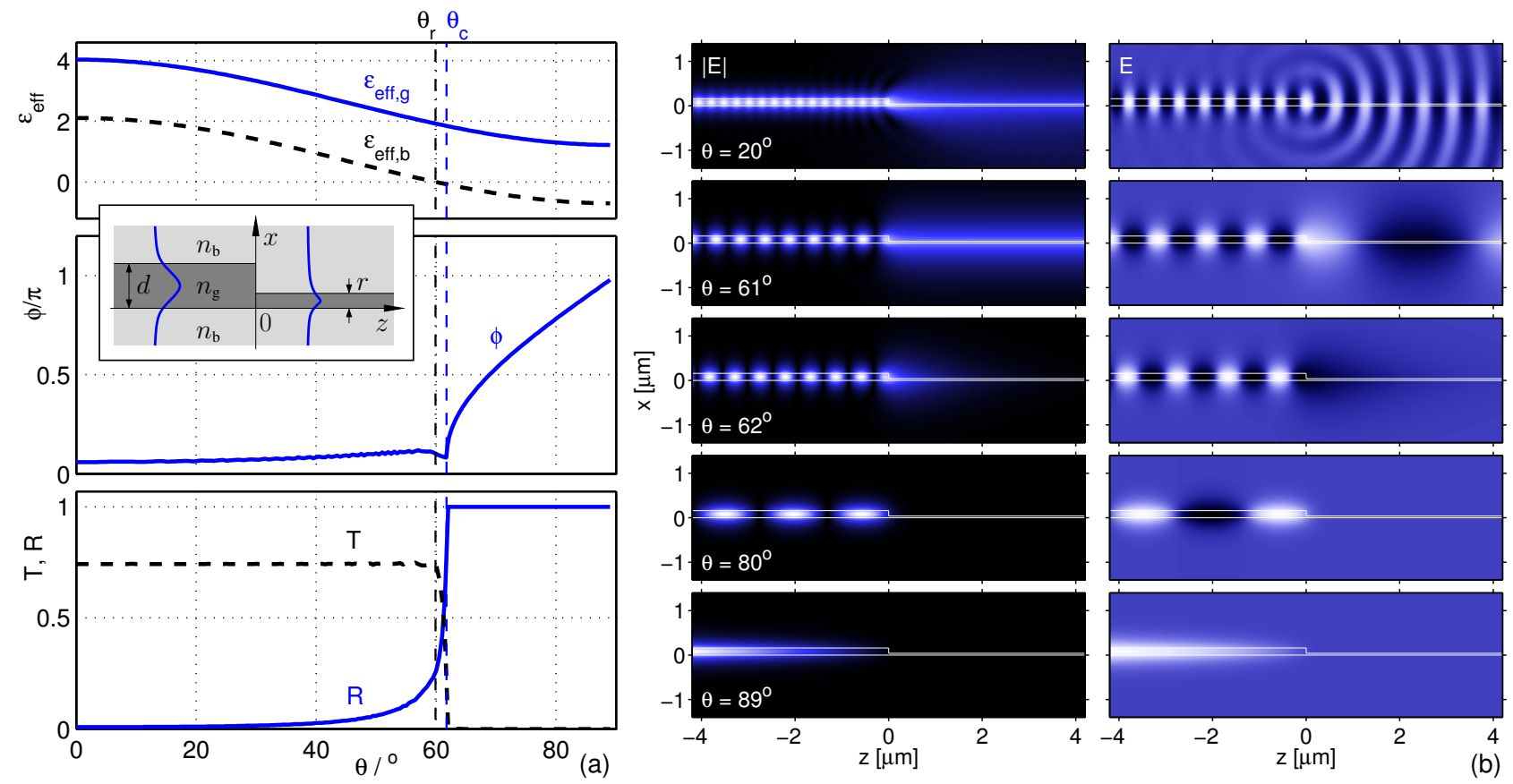

Figure 6: Reflection of a semi-guided plane wave at a step discontinuity. (a): Guided wave reflectance $R$, transmittance $T$, phase change upon reflection $\phi$, and effective permittivities of the background $\epsilon_{\mathrm{eff}, b}$ and guiding regions $\epsilon_{\mathrm{eff}, \mathrm{g}}$, versus the angle of incidence $\theta$. (b): absolute values and time snapshots of the time-harmonic scalar field $E$ associated with the effective problem (12) for different angles of incidence $\theta$. 
At $\theta=\theta_{\mathrm{r}}$, the effective permittivity $\epsilon_{\mathrm{eff}, \mathrm{b}}$ associated with the substrate and cladding regions, becomes negative. This manifests as well if one takes a look at the associated fields in Figure 6(b). At $\theta=20^{\circ}<\theta_{\mathrm{r}}<\theta_{\mathrm{c}}$, backwards and forward traveling propagating waves are visible, corresponding to forwards and backwards radiative losses. These waves are suppressed for $\theta_{\mathrm{r}}<\theta=61^{\circ}<\theta_{\mathrm{c}}$; the field is to be attributed mainly to the fundamental modes, with a partly standing, partly traveling wave in the input segment $z<0$, and the wider, outgoing guided mode for $z>0$. At even higher angles of incidence $\theta_{\mathrm{r}}<\theta_{\mathrm{c}}<\theta=62^{\circ}, 80^{\circ}, 89^{\circ}$, the incident guided mode is being fully reflected. No propagating waves are permitted in region (II); for $z>0$ one merely observes evanescent field tails that decrease in extension, if $\theta$ grows towards grazing incidence.
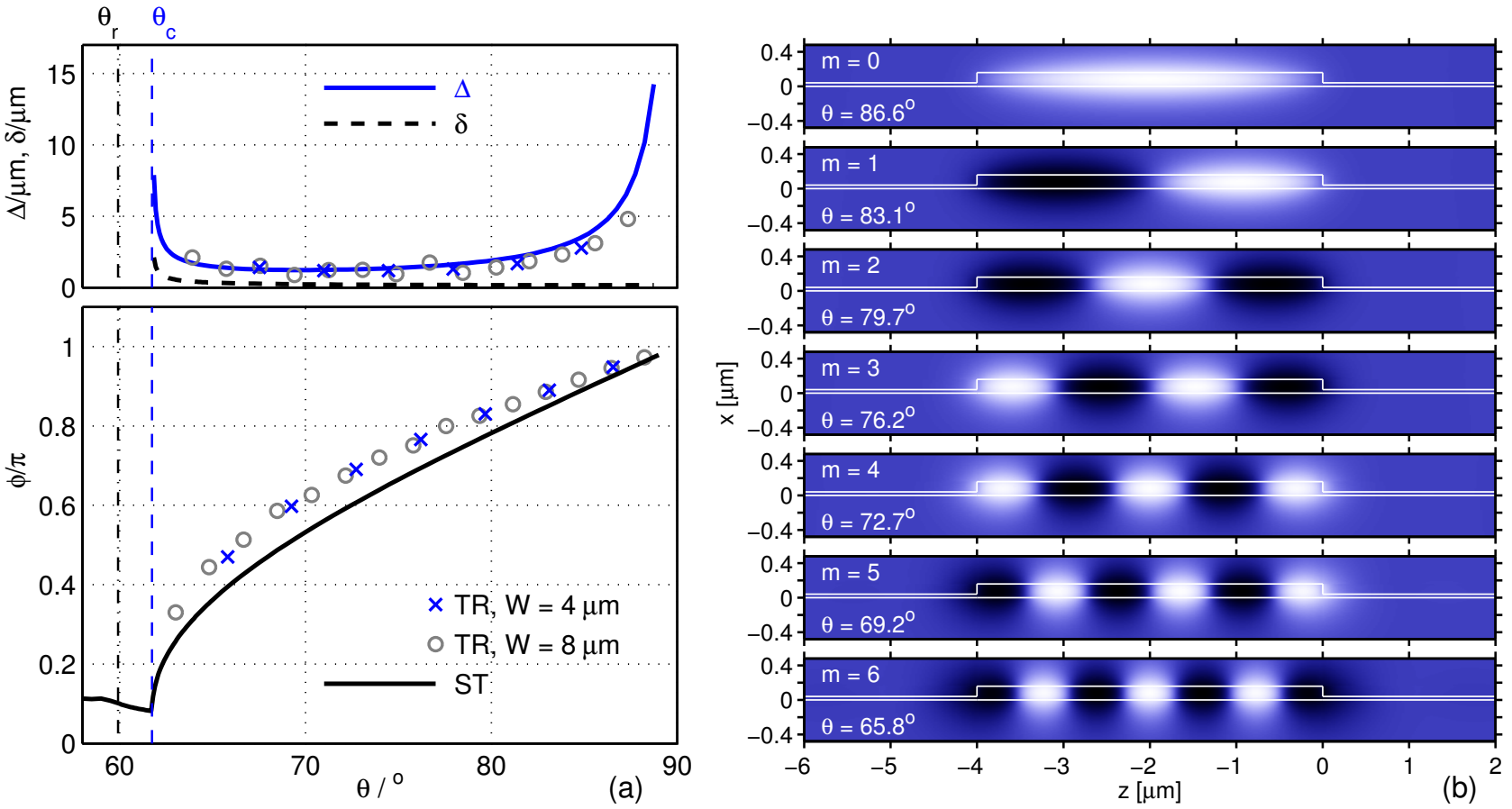

Figure 7: Total internal reflection of semi-guided plane waves at the step discontinuity of Figure6. (a): Phase change $\phi$ of the guided wave upon reflection, associated Goos-Hänchen-shift $\Delta$, and the effective boundary distance $\delta$, as a function of the angle of incidence $\theta$; estimates determined as outlined in Section 3 (scalar theory, ST) and Section 4 (transverse resonance, TR), in the later case by mode analysis of rib waveguides of different widths $W$. (b) Guided mode profiles of a rib of width $W=4 \mu \mathrm{m}$, constituted by two of the former step discontinuities, with associated mode indices $m$ and mode angles $\theta$.

Figure 7 considers the range of angles with total internal reflection in more detail. In part (a) the $\phi(\theta)$-curve of Figure 6(a) is being accompanied by values from mode calculations for channel waveguides of different widths. Some of these mode profiles, together with mode indices and mode angles, are shown in Figure 7 (b). The profiles relate to the fields of Figure 6(b), for nearby angles of incidence.

For grazing incidence $\theta \rightarrow 90^{\circ}$, one expects the field at the interface $z=0$ to vanish; accordingly the phase change upon reflection $\phi$ approaches $\pi$ (cf. Eq. (13)). $\phi$ decreases for lower incidence angles, with growing slope, down to the kink at the critical angle $\theta_{c}$. By using finite-difference approximations for the derivatives, the $\phi(\theta)$-data can be translated to the curves for the beam displacement $\Delta$ and the effective boundary position $\delta$, as shown in the upper panel of Figure 7 (a). Large values for the Goos-Hänchen-shift emerge for the steep slope of $\phi(\theta)$ at the critical angle, and for vanishing $\cos \theta$ for grazing incidence. The geometrical "penetration depth" of the beams remains small for the step transitions; the largest values are found for the long evanescent field tails just above the critical angle.

\subsection{Tapered transition}

Results for linearly tapered transitions have been collected in Figure 8. For part (a) the parameters are as given in Figures 6, 7, the curves in those figures can thus be viewed as the limit of the data in Figure 8 for zero taper length. To match the actual final design in Ref. [9], for the simulations in (b) we assumed a slightly larger thickness $d=170 \mathrm{~nm}$, leading to accordingly different angles $\theta_{\mathrm{r}}=59.08^{\circ}$ and $\theta_{\mathrm{c}}=60.84^{\circ}$. Nevertheless the curves in Figure 8(b) should be discussed as part of the series in Figure 8 (a), for a "long" taper. 

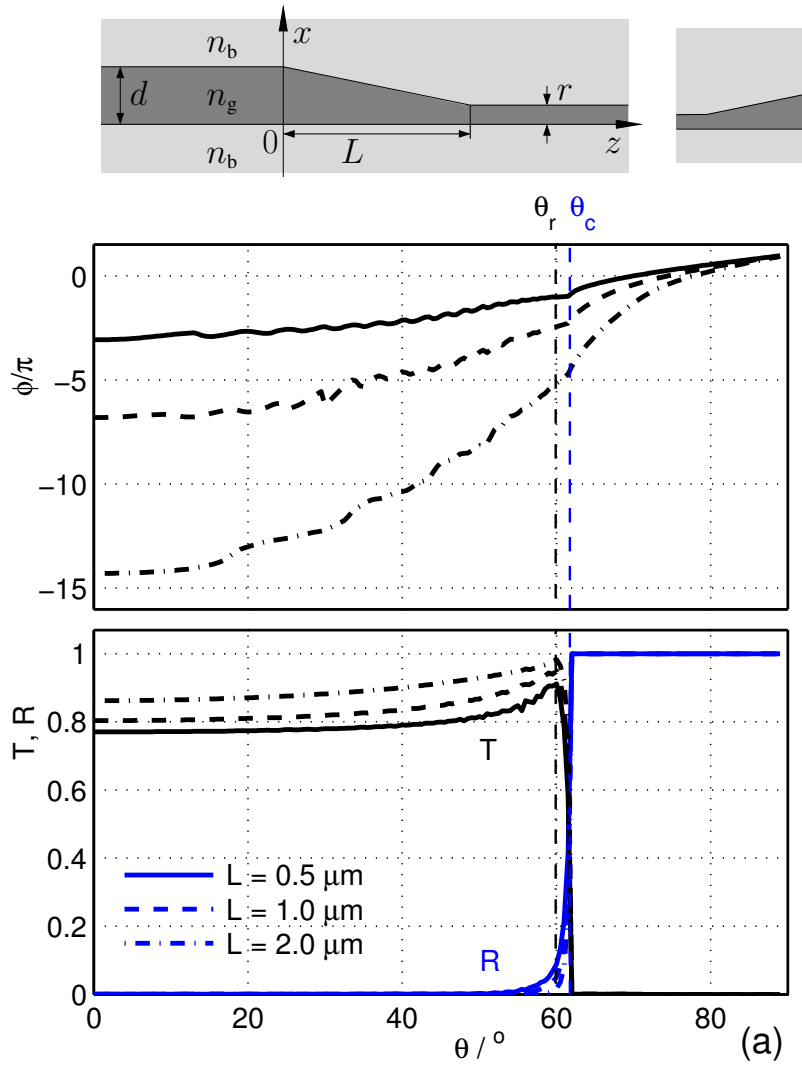

\section{$W$}
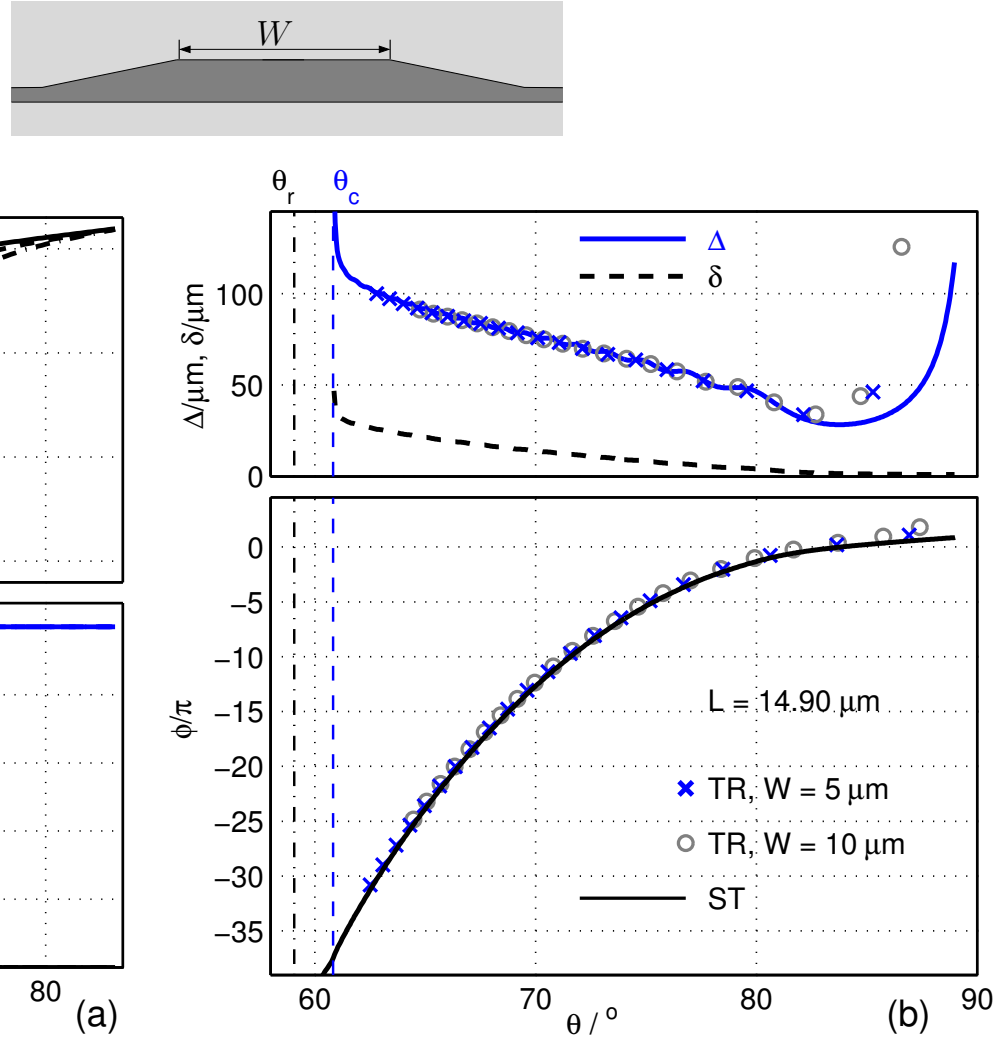

Figure 8: Simulations of tapered transitions of different length $L$. (a): Reflectance $R$, transmittance $T$, and the phase change upon reflection $\phi$ as a function of the angle of incidence $\theta$, computed with the scalar approach of Section 3 . (b): Configurations with total internal reflection, phase change upon reflection $\phi$, lateral beam shift $\Delta$ and geometrical penetration depth $\delta$ versus the incidence angle $\theta$, for a taper extension $L=14.90 \mu \mathrm{m}\left(\right.$ taper angle $\left.0.5^{\circ}\right)$.

The effects of lossless total internal reflection beyond a critical angle of incidence, and the partial lossless reflection in a small intermediate interval of incidence angles, occur for the tapers as well as for the step discontinuity. Note that these characteristic angles do not depend on the intermediate shape of the transition.

The absolute phase change upon reflection grows with increased taper length, as does the lateral beam displacement, and the penetration depth of the beam into the tapered region. The kink in the $\phi(\theta)$-curve at $\theta=\theta_{c}$ appears to become less pronounced for more extended transitions. Just as for the step discontinuities, extremal values for $\Delta$ and $\delta$ are observed for incidence close to the critical angle, and for grazing incidence. Still, in case of the long taper of Figure 8(b), these deviations are substantial also for intermediate angles reasonably well above the critical angle, e.g. at $\theta=65^{\circ}$ with $\Delta=92 \mu \mathrm{m}$ and $\delta=21 \mu \mathrm{m}$. Here the geometrical penetration depth turns out to be larger than the actual length of the taper.

\section{Concluding remarks}

Standard simulation tools can provide approximate quantitative insight on the 3-D slab-transition problem. Where applicable, the results obtained with a scalar 2-D Helmholtz solver and by guided mode analysis of rib waveguides with 2-D cross sections agree reasonably well, given the underlying approximations. Both scalar approaches take the light polarization into account only through the vertical shapes of the major parts of the optical fields, which are here assumed to be in-plane polarized.

Two characteristic angles have been identified, determined solely by the properties of the input- and output regions. Radiation losses are forbidden for wave incidence beyond the first angle, while guided transmission is still allowed. For incidence at angles larger than the second, the critical angle, the entire incident power is being reflected into the guided incoming mode. Only in that regime the "conventional" viewpoint, where one assigns effective mode indices to the regions with constant thickness, followed by application of Snell's law, is valid. These observations hold for step discontinuities as well as for tapered transitions of (reasonably) arbitrary shape and extension, exemplified by our results on linear tapers of different lengths. 
More accurate results would require the (computational) solution of the exact equations. This then concerns a vectorial Helmholtz equation on a 2-D computational domain, with transparent boundary conditions that permit the influx of the properly rotated guided slab mode. Since formally the problem is identical to a standard vectorial mode eigenvalue problem, it should be possible to modify some suitable solver accordingly. It is to be anticipated that phenomena like polarization coupling / field hybridization, as found for specific channel waveguides, will also occur for the present angled slab transition problems.

\section{Acknowledgments}

This research is supported by the Dutch Technology Foundation STW (project 10051). The authors thank R. Stoffer and M. Maksimovic for many fruitful discussions.

\section{References}

[1] P. K. Tien. Integrated optics and new wave phenomena in optical waveguides. Reviews of Modern Physics, 49(2):361-419, 1977.

[2] R. Ulrich and R. J. Martin. Geometrical optics in thin film light guides. Applied Optics, 10(9):2077-2085, 1971.

[3] G. C. Righini, V. Russo, S. Sottini, and G. Toraldo di Francia. Geodesic lenses for guided optical waves. Applied Optics, 12(7):1477-1481, 1973.

[4] F. Zernike. Luneburg lens for optical waveguide use. Optics Communications, 12(4):379-381, 1974.

[5] S. Misawa, M. Aoki, S. Fujita, A. Takaura, T. Kihara, K. Yokomori, and H. Funato. Focusing waveguide mirror with a tapered edge. Applied Optics, 33(16):3365-3370, 1994.

[6] C.-C. Tseng, W.-T. Tsang, and S. Wang. A thin-film prism as a beam separator for multimode guided waves in integrated optics. Optics Communications, 13(3):342-346, 1975.

[7] G. C. Righini and G. Molesini. Design of optical-waveguide homogeneous refracting lenses. Applied Optics, 27(20):4193-4199, 1988.

[8] F. Çivitci and H. J. W. M. Hoekstra. Design of spectrometers and polarization splitters using adiabatically connected slab waveguides. In 16th European Conference on Integrated Optics, ECIO 2012, Sitges/Barcelona, Spain, page Paper 151, 2012.

[9] F. Çivitci, M. Hammer, and H. J. W. M. Hoekstra. Design of a prism spectrometer based on adiabatically connected waveguiding slabs, Journal of Lightwave Technology (submitted for publication, 2013).

[10] H. K. V. Lotsch. Reflection and refraction of a beam of light at a plane interface. Journal of the Optical Society of America, 58(4):551-561, 1968.

[11] A. W. Snyder and J. D. Love. Optical Waveguide Theory. Chapman and Hall, London, New York, 1983.

[12] M. Hammer and O. V. Ivanova. Effective index approximations of photonic crystal slabs: a 2-to-1-D assessment. Optical and Quantum Electronics, 41(4):267-283, 2009.

[13] M. Born and E. Wolf. Principles of Optics, 7th. ed. Cambridge University Press, Cambridge, UK, 1999.

[14] A. S. Sudbø. Film mode matching: a versatile numerical method for vector mode fields calculations in dielectric waveguides. Pure and Applied Optics, 2:211-233, 1993.

[15] FIMMWAVE - Waveguide Mode Solvers. Photon Design, 34 Leopold Street, Oxford OX4 1TW, United Kingdom; http://www.photond.com/

[16] FieldDesigner, advanced optical mode solvers. PhoeniX Software, P.O. Box 545, 7500 AM Enschede, The Netherlands; http://www.phoenixbv.com/

[17] F. Çivitci, M. Hammer, and H. J. W. M. Hoekstra. Reflection of semi-guided plane waves at angled thin-film transitions. XXI International Workshop on Optical Wave \& Waveguide Theory and Numerical Modeling, Enschede, The Netherlands, 2013.

[18] C. Vassallo. Optical Waveguide Concepts. Elsevier, Amsterdam, 1991.

[19] C. Vassallo. 1993-1995 Optical mode solvers. Optical and Quantum Electronics, 29:95-114, 1997.

[20] M. Hammer. Quadridirectional eigenmode expansion scheme for 2-D modeling of wave propagation in integrated optics. Optics Communications, 235(4-6):285-303, 2004. 
[21] M. Hammer. METRIC - Mode expansion tools for 2D rectangular integrated optical circuits. http://www.math.utwente.nl/ hammerm/Metric/

[22] M. Lohmeyer. Wave-matching method for mode analysis of dielectric waveguides. Optical and Quantum Electronics, 29:907-922, 1997. 